\title{
SIMULATIONS AND MEASUREMENTS OF A HEAVILY HOM-DAMPED MULTI-CELL SRF CAVITY*
}

\author{
Haipeng Wang ${ }^{\#}$, Robert Rimmer, Frank Marhauser, TJNAF, Newport News, VA, 23606
}

\section{Abstract}

After an initial cavity shape optimization [1] and cryomodule development [2] for an Ampere-class FEL ERL, we have simulated a complete 5-cell high-current (HC) cavity structure with six waveguide (WG) couplers for Higher Order Mode (HOM) damping and fundamental power coupling. The time-domain wakefield simulations of the MAFIA codes have been used to calculate the cavities broadband HOM impedance spectrum. Microwave Studio (MWS) has also been used to evaluate the external $\mathrm{Q}$ of the fundamental power coupler (FPC) and the R/Qs of the HOMs. A half scale $1497 \mathrm{MHz}$ singlecell model cavity and a 5-cell copper cavity including dummy HOM WG loads were fabricated to bench measure and confirm the design performance. Details of the multi-beam wakefield simulations, the HOM damping measurements and multi-peak data fitting analysis techniques are presented.

\section{CAVITY DESIGN}

The optimized high current $748.5 \mathrm{MHz}$ cavity center cell shape exhibits a comparably large beam iris yielding a small loss factor $\mathrm{k}$ and an efficient HOM damping. The equator outline starts with a flat wall joining a shallow dome towards a "low loss" concept (high $R / Q^{*} G$ ) [1]. Figure 1 shows our final design geometry.

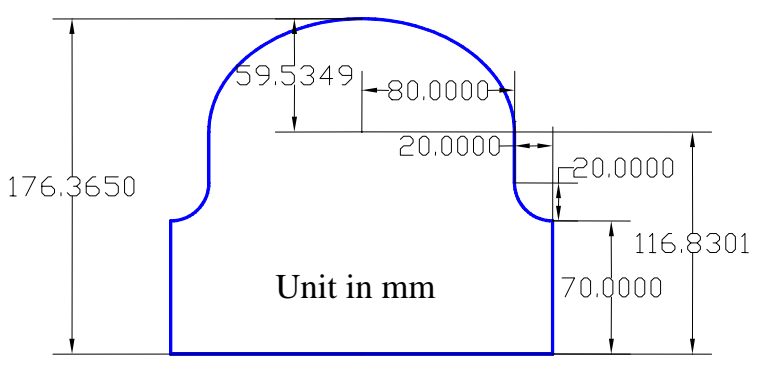

Figure 1: Center cell shape for the 748.5 MHz HC cavity.

Table 2: 5-cell, HC 748.5MHz cavity design parameters.

\begin{tabular}{|l|l|l|l|}
\hline $\mathrm{R} / \mathrm{Q}=\mathrm{V}_{\mathrm{acc}}{ }^{2} /(\omega \mathrm{U})$ & $525 \Omega$ & Geo. Factor & $276 \Omega$ \\
\hline $\mathrm{E}_{\text {peak }} / \mathrm{E}_{\mathrm{acc}}$ & 2.50 & $\mathrm{k}_{\mathrm{cc}}$ cell-to-cell & $3.26 \%$ \\
\hline $\mathrm{B}_{\text {peak }} / \mathrm{E}_{\mathrm{acc}}$ & $4.27 \mathrm{mT}$ & $\mathrm{k}_{/ /}$for $\sigma_{\mathrm{z}}=1 \mathrm{~mm}$ & $5.6 \mathrm{~V} / \mathrm{pC}$ \\
\cline { 5 - 5 } & $/(\mathrm{MV} / \mathrm{m})$ & $\mathrm{k}_{\perp}$ for $\sigma_{\mathrm{z}}=1 \mathrm{~mm}$ & $2.95 \mathrm{~V} / \mathrm{pC} / \mathrm{m}$ \\
\hline
\end{tabular}

The parameters in Table 2 have been obtained from $2 \mathrm{D}$ simulations using the SuperFish and ABCI94 codes. We adopted end-cell shape being equal to the center-cell,

\footnotetext{
* Authored by Jefferson Science Associates, LLC under U.S. DOE Contract No. DE-AC05-06OR23177 and by The Office of Naval

Research under contract to the Department of Energy.

\# E-mail: haipeng@jlab.org
}

however the end-cells are trimmed at equator (shorter by $2.1 \%$ of the fundamental wavelength) to attain a flat field profile along the cavity. As a benefit, the electron beam can utilize the fringe fields in the beam pipe region. Thus the $\mathrm{R} / \mathrm{Q}$ could be enhanced by $\sim 5 \%$ compared to the case when the end cells are different in shape but equal in length compared to the center cells. Only a single die model is needed for the cavity production. We have made a half-scale $1497 \mathrm{MHz}$ copper prototype and two production $\mathrm{Nb}$ cavities. Bead-pull measurements indicate that with a minor tuning, the field flatness can be $99 \%$ without resembling a "reentrant" shape. The simulation shows no more field enhancement at the end-cells as well. The cavity cold test results and overall progress of this project can be found in references [3, 4].

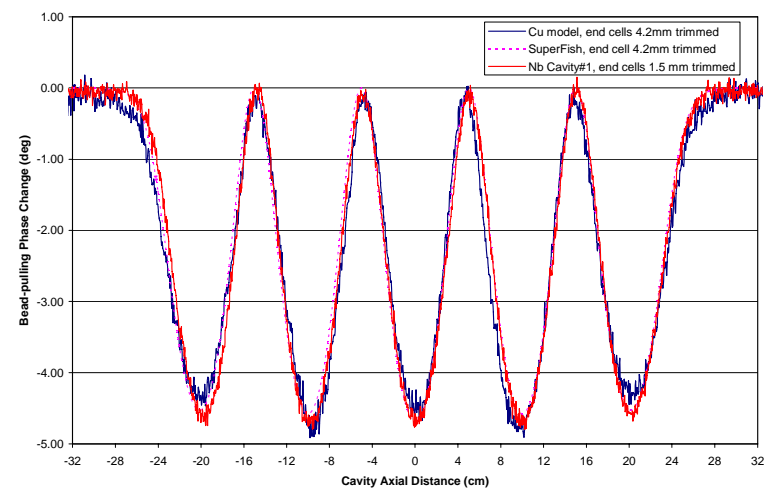

Figure 2: On-axis E-field flatness, simulation verses beadpull measurement for $1497 \mathrm{MHz} \mathrm{Cu}$ and $\mathrm{Nb}$ cavities.

\section{FUNDAMENTAL POWER COUPLER}

The HC cavity uses one of six rectangular WGs $(90 \mathrm{~mm} \times 41.3 \mathrm{~mm})$ as the FPC. The FPC steps up in width and tapers up in height into a standard WR650 towards the RF source. The step distance $d$ to the beam line center (length of below-cutoff WG) determines the coupling $\mathrm{Q}_{\text {ext }}$. We used MWS to calculate $\mathrm{Q}_{\text {ext }}$. The build-in macro for $\mathrm{Q}_{\mathrm{ext}}$ in the eigen solver uses a modified Balleyguier's method [5]. We found the $d=348.3 \mathrm{~mm}$ for the $\mathrm{Q}_{\text {ext }}$ spec of $3.2 \times 10^{6}$, on the $748.5 \mathrm{MHz}$ cavity. However for the 1497 $\mathrm{MHz}$ cavity to be used at the FEL injector, the $\mathrm{Q}_{\text {ext }}$ spec is $9.5 \times 10^{5}$ due to the beam loading. In order to avoid the step being too close to the cavity, a pair of ridges is used in the WG to enhance the coupling field. This results in a reasonable $d=180 \mathrm{~mm}$. A bench measurement on the copper model agreed with the MWS calculation and tolerable error analysis (Figure 3). When using a transmission method to measure the $\mathrm{Q}_{\text {ext }}$ of the FPC as shown in Figure 4, a special calibration procedure called "adapter removal" has to be applied on an Agilent 8753ES VNA in order to achieve the desired accuracy. 


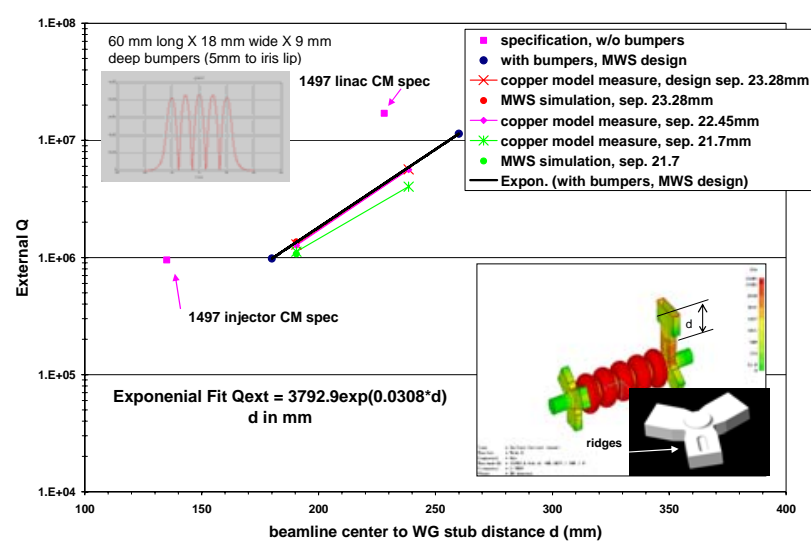

Figure 3: FPC $\mathrm{Q}_{\text {ext }}$ simulation and measurement data.

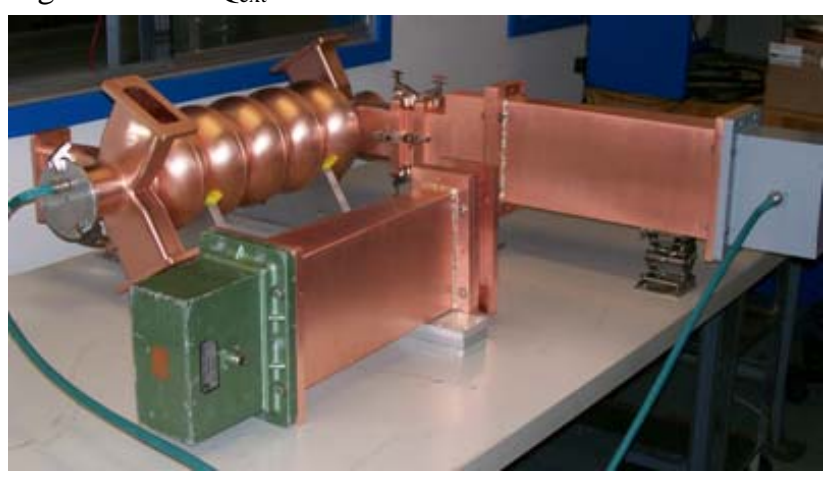

Figure 4: Setup of the 1497MHz 5-cell Cu cavity prepared for the FPC $\mathrm{Q}_{\mathrm{ext}}$ measurements.

At high beam currents, one concern is a considerable charging of the vacuum RF window due to image currents if the window is in line-of sight with the electron beam. A major design goal was to optimize a "dogleg" transition to the circular RF window (warm), which is centered in an iris flanged to the standard sized WR650 shoulders (see Figure 5). Corresponding MWS simulations indicated that with an unmatched transition assembly, the $\mathrm{Q}_{\text {ext }}$ can alter by more than $25 \%$ due to either the offset of the notch frequency and/or a shallow reflection response of $|\mathrm{S} 11|$. The notch frequency is very sensitive to the dielectric constant and the position of the ceramic with respect to the iris center. Moreover, the dip of $|\mathrm{S} 11|$ strongly depends on the tapered "dogleg" outline. The transition assembly actually becomes a rather narrowband device. However, an optimized "dogleg" shape can yield a $|\mathrm{S} 11|$ of $-30 \mathrm{~dB}$ within a $\pm 10 \mathrm{MHz}$ bandwidth keeping the original $\mathrm{Q}_{\mathrm{ext}}$ unchanged.
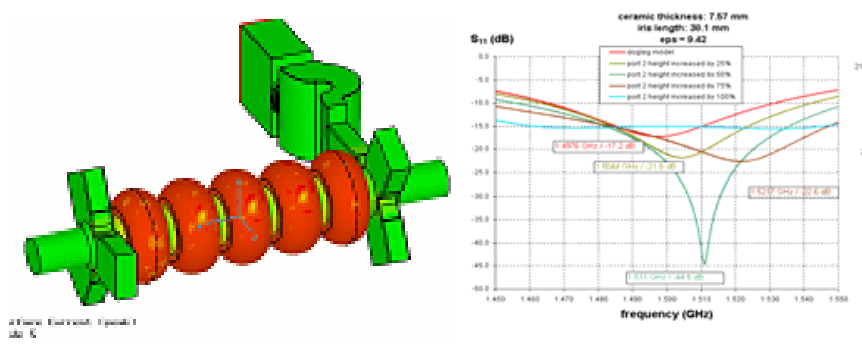

Figure 5: FPC transition match simulation by MWS.

\section{HIGH POWER HOM LOADS}

Each of five HOM absorbers has to take $4 \mathrm{~kW}$ of HOM power determined by the HOM narrowband and broadband calculations. The RF design used the MWS transient solver. Measured complex permittivity data of the $\mathrm{SiC}$ material from one vendor were used for the $2^{\text {nd }}$ order Debye function fitting in the MWS. The WG dimensions and absorber wedge shapes were optimized to attain the $|\mathrm{S} 11|<0.2$ from 1 to $8 \mathrm{GHz}$ (Figure 6). The RF field details have also been benchmarked with ANSYS for a further RF-thermal coupled analysis [6]. The bench measurement for a 40W HOM load derived from the same design is under the way.

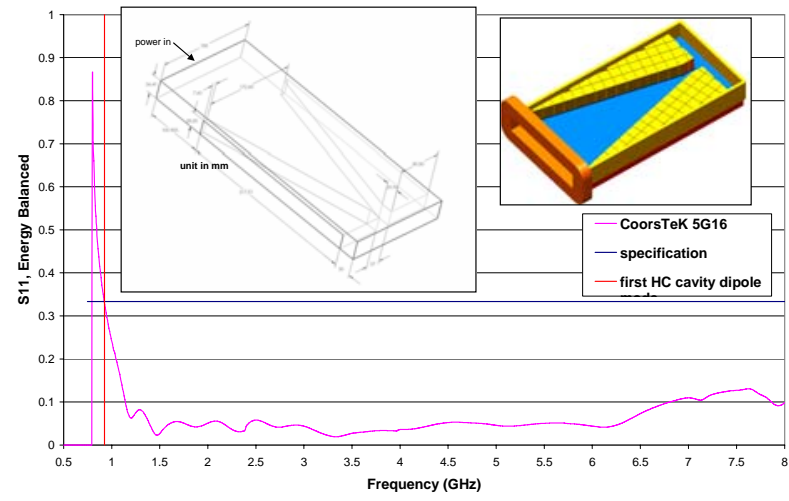

Figure 6: MWS design of 4kW broadband HOM load.

\section{HOM DAMPING SIMULATIONS}

The powerful method to derive the broadband impedance spectrum of a heavily HOM-damped cavity is using the MAFIA 3D time domain capability. Both broad and narrow band HOM impedances and loaded Qs (except the unresolved TM010 modes) can be obtained if the beam induced wake potential is monitored and a proper FFT and normalization is done. The details of the simulation and post processing procedure we used are summarized briefly in the following:

1. Generate 3D cavity vacuum CAD drawing with WG ports facing to Cartesian coordinates.

2. Import CAD geometry. Mesh the volume with uniform steps $(\sim 2.5 \mathrm{~mm})$ in beam direction.

3. Define WG and beam port positions and mesh them in 2D. Solve WG modes in 2D.

4. Load WG solutions to each port with zero power input. Check any flag on ignored mode.

5. Set up "1D current" in Gaussian bunch with $\mathrm{s}=3 \mathrm{~cm}, 0 \sim 10 \sigma$ long at the defined off-axis distances. Total source and image charges $=1 \mathrm{C}$.

6. Set up wake potential monitors in the beam direction at the defined polarization paths.

7. Calculate $\sim 672 \mathrm{~m}$ long wake potential for $\sim 2$ million meshes. It takes $\sim 6$ days for each run.

8. Take max. $2^{\mathrm{n}}$ numbers of wake data set with a Cosine window tapering ( $\mathrm{n}=18$ for Figure 7 ).

9. FFT the wake $\mathrm{W}_{\mathrm{zfft}}$ and Gaussian beam B. 
10. Normalize impedance with $\mathrm{Z}_{\mathrm{am}}=\left|\mathrm{W}_{\mathrm{zfft}}\right| \mathrm{B}_{\mathrm{am}}$ and $\mathrm{Z}_{\mathrm{ph}}=\mathrm{W}_{\mathrm{zftph}}-\mathrm{B}_{\mathrm{ph}}$. With the correction of phase wrapping within $-90^{\circ}$ to $90^{\circ}$, the real part of impedance $\mathrm{Z}_{\mathrm{re}}$ should be always $>0$.

A special MathCAD program has been developed for data analysis and processing. Due to a mesh-size related instability in the time domain algorithm and computer power limitations, only up to $8 \mathrm{GHz}$ of the $\mathrm{HOM}$ spectrum has been evaluated.

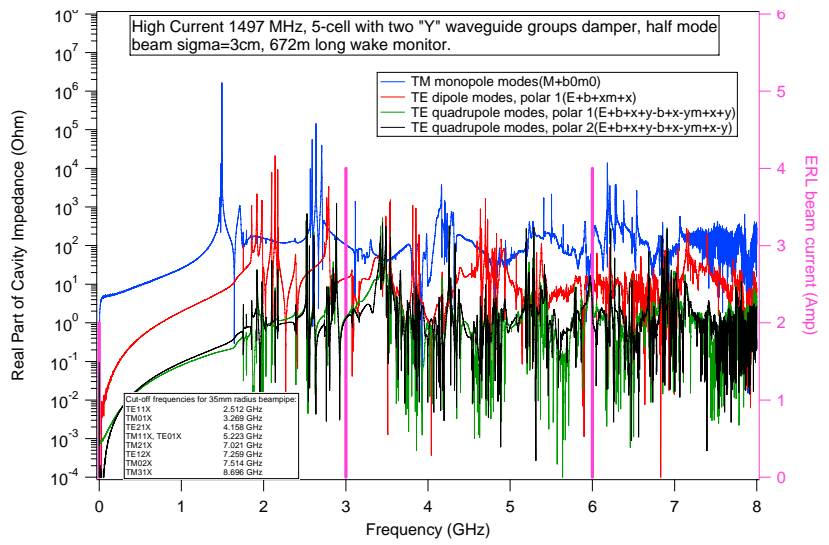

Figure 7: HOM spectra calculated utilizing "multi-beam" excited wakefield schemes in MAFIA 3D, T3 solver.

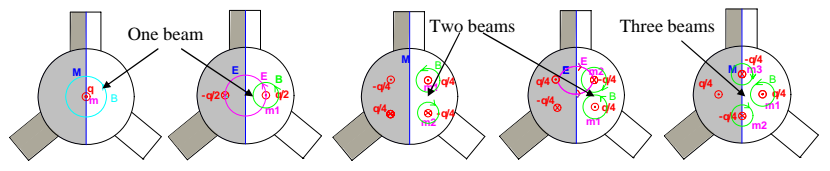

Monopole modes Dipole modes, polar 1 Dipole modes, polar 2 Quad modes, polar 2 Quad modes, polar 1 Figure 8: "Multi-beam" wake excitation scheme as used in the MAFIA wakefield simulations, $\mathrm{E}$ denotes the electric, and $\mathrm{M}$ the magnetic symmetry boundary. $\mathrm{m} 1$, or $\mathrm{m} 2$ is the wake polarization monitor set in the path.

It is particularly difficult to identify the mode type, i.e. TM/TE monopole, dipole etc. in a well-damped multi-cell cavity since mode overlapping is prevalent. In order to identify the HOMs in a broadband spectrum where the tails of multiple modes are mixed, we have developed a co-moving "multi-beam" wake excitation method which can effectively suppress unwanted mode types. Figure 8 illustrates these applied schemes. For each setup, a particular mode type as monopole, dipole, quadruple or even higher type can be extracted purely. Their overall impedance values can be classified by two orders of amplitude. We used two current lines (in Figure 7) of the ERL beam to calculate the monopole and dipole modes deposited energy in the $748.5 \mathrm{MHz}$ cavity below cutoffs.

\section{HOM LOADED Q MEASUREMENT}

A single-cell aluminum model with one WG end group and a whole 5-cell copper cavity have been fabricated to verify the calculated HOM impedances and loaded Qs. The R/Q of each trapped mode can be obtained either from a "bead-pull" measurement or simulations (2D or 3D). The loaded Qs were measured on bench taking into account the loss contribution from the material and the coupling strength of the used probes. Alternatively the cavity resonance spectrum measured with two weakly coupled beam pipe antennas have been numerically fitted to evaluate the loaded Qs in case of strong mode overlap. Therefore an available fitting program [7] has been further modified to find the real amplitudes of the resonances and filter out the contributions from up to four neighboring modes including and background signals. This fitting program can be also used to find the real impedance of each HOM in the impedance amplitude spectra obtained from MAFIA and be readily compared to Figure 7 . Measured data for the $\mathrm{Q}_{\text {load }}$ combined with the R/Qs of MAFIA 2D eigenmode results for the single cell cavity structure yields impedances are in good agreement with the impedance simulations, particularly for those modes below the cutoffs. Using the MWS 3D eigenmode solver $\mathrm{R} / \mathrm{Qs}$ gives even more accurate results. This has been confirmed by bead-pull measurements. As an example, Figure 9 shows the calculated dipole mode spectra together with the measured impedances of the most parasitic dipole HOMs.

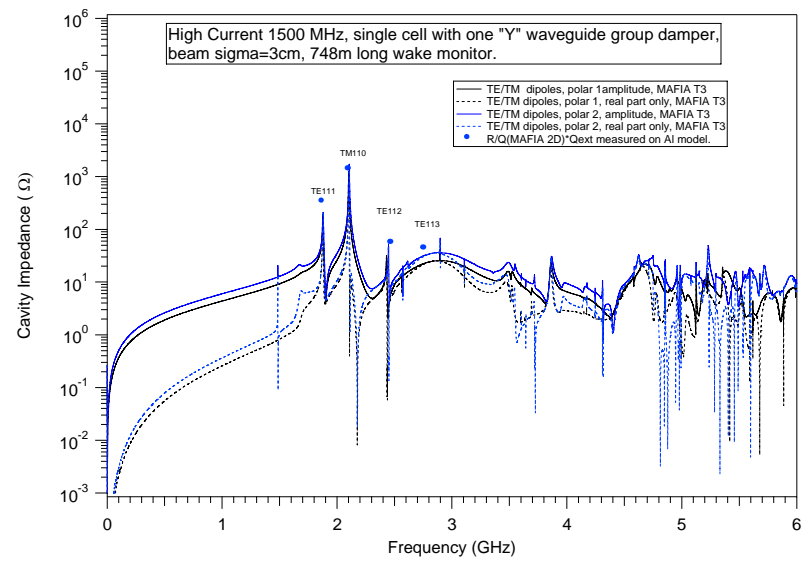

Figure 9: HOM dipole impedance on a single-cell HC aluminum model, simulation verses measurement.

\section{SUMMARY}

We have designed a scheme for heavily HOM damped superconducting cavities with various simulation tools. Efforts to verify the measurement data have been done for a single cell cavity. So far the simulations and measurements are in excellent agreement. A five-cell cavity is currently under the measurement data analysis to demonstrate HOM-damping capability towards the needs of an Ampere-class FEL ERL.

\section{REFERENCES}

[1] H. Wang et. al., PAC’ 2005, Knoxville TN, p. 4191. [2] R. A. Rimmer et al., EPAC’ 2006, Edinburgh, p. 490. [3] P. Kneisel et al., WEPMS063, these proceedings. [4] R. A. Rimmer et al., WEPMS068, these proceedings. [5] V. Shemelin et.al, Cornell Univ. SRF note 020620-03. [6] G. Cheng et al., MOPAS075, these proceedings.

[7] D. A. Goldberg et al., LBNL Report-40290. 